\title{
ENFERMERÍA
}

\section{Patrones de demandas de autocuidado en estudiantes universitarios de la carrera de Enfermería de la Universidad Nacional de Canindeyú, sede Salto del Guairá}

\author{
Bruna Vera ${ }^{1}$, Raquel Villalba ${ }^{1}$, Teresa Villalba ${ }^{1}$, Andrea Acosta ${ }^{1}$
}

\section{Resumen:}

Introducción: La Teoría General del Déficit de Autocuidado de Dorothea Orem tiene fuerte repercusión internacional en el ámbito de la enfermería, por su propiedad para el diseño de currículos así como por los diversos estudios de campo que la validaron. El desarrollo de la Enfermería, ha generado la construcción de modelos que constituyen un conjunto de conceptos abstractos y generales desarrollados a partir de la observación empírica y la deducción o inducción desde los campos de investigación. Cada modelo conceptual se constituye en una guía teórica que orienta la práctica de Enfermería, fomenta el conocimiento y estimula la Investigación. En el transcurso de los años, los modelos de cuidado de enfermería se han ido empleando y perfeccionando hasta llegar a tener la solidez de la teoría. Y han hecho crecer la enfermería como ciencia, disciplina y profesión. Entre las teóricas más influyentes de la enfermería se destaca la estadounidense Dorothea Elizabeth Orem. Ella propone tres teorías relacionadas entre sí, todas con la finalidad de mantener la funcionalidad vital del individuo mediante el cuidado que la persona realiza de sí misma. La teoría del autocuidado, la teoría del déficit del autocuidado, la teoría de sistemas de enfermería, juntas, constituyen una teoría general de enfermería, denominada Teoría de enfermería del déficit de autocuidado.

Objetivo: Analizar patrones de demandas de autocuidado según requisitos universales, en los estudiantes de la carrera de Enfermería de la Universidad Nacional de Canindeyú, sede Salto del Guairá.

Métodos: Se condujo un estudio cualitativo, de corte etnográfico. Los datos se recogieron por medio de entrevistas semiestructuradas, aplicadas a 27 estudiantes de la población de interés, explorando patrones de demandas asociadas a los 8 Requisitos Universales formulados por Orem. El muestreo fue teórico. Se construyó la muestra con el criterio de la mayor variabilidad posible.

1. Universidad Nacional de Canindeyú, Facultad de Ciencias de la Salud, Paraguay.

E-mail: bruhsheeran43@gmail.com

DOI: 10.26885/rcei.foro.2019.221

Trabajo publicado en acceso abierto bajo Licencia Creative Commons. 
Previo consentimiento informado, se tomaron datos sociodemográficos. Se les pidió, luego, permiso, a los entrevistados, para grabar la entrevista. Lograda la autorización, se les pidió relatar su rutina diaria con el mayor detalle posible. A partir de las respuestas se profundizaron en determinadas líneas, por medio de repreguntas. Con las grabaciones de las entrevistas se constituyeron los documentos primarios que pasaron a formar parte de la unidad hermenéutica. Se identificaron y codificaron citas relacionadas con las demandas de autocuidado vinculadas con los requisitos universales. Con las demandas identificadas se graficaron redes semánticas.

Resultados: Los datos analizados muestran demandas frecuentes asociadas con 5 de los requisitos universales. Las redes semánticas objetivadas sugieren relaciones causales entre demandas. Al producirse desequilibrios en un requisito, se producen demandas en otros, como en cascada. Se observan casos de hasta 5 demandas simultáneas, en asociación con variables sociodemográficas, predichas en la teoría.

Conclusión: Los resultados sugieren que, de no tomarse acciones de prevención con el sistema de enfermería de apoyo educacional, en el mediano plazo muchas personas de la población estudiada desarrollarán enfermedades crónicas relacionadas con los desequilibrios de las demandas identificadas en los requisitos.

Palabras clave: autocuidado, demandas de autocuidado, requisitos universales.

\section{RefERENCIAS}

Orem, D. E. (1993). Modelo de Orem: Conceptos de Enfermería en la práctica. Barcelona, España: Masson-Salvat.

Pereda Acosta, M. (2011). Explorando la teoría general de enfermería de Orem. Enf Neurol., 10(3), 163-7.

Ruedas Nuñez, Y. M., Gálvez Días, N del C. (2013). Estilos de autocuidado de estudiantes de enfermería en el contexto universitario: Un análisis desde la teoría de Dorothea Orem, Chiclayo, 2013. Tzhoecoen, 6(1), 241-52.

Sánchez Rueda, G., Dorothea, E. (1999). Orem Aproximación a su teoría. Rev ROL Enf., 22(4), 309-11.

Vega Angarita, O. M, González Escobar, S. D. (2007). Teoría del déficit de autocuidado: interpretación desde los elementos conceptuales. Rev Cienc y Cuid., 4(1), 28-35. 